\title{
A Graphical Interval Logic Toolset for Verifying Concurrent Systems ${ }^{\star}$
}

\author{
G. Kutty, Y. S. Ramakrishna, L. E. Moser, L. K. Dillon, P. M. Melliar-Smith ${ }^{\star \star}$ \\ Departments of Electrical and Computer Engineering \\ and of Computer Science \\ University of California, Santa Barbara
}

\begin{abstract}
Graphical Interval Logic is the foundation of a toolset we have developed to support formal specification and verification of concurrent systems. The logic is a discrete linear-time temporal logic with the distinguishing feature that formulas in the logic have an intuitive graphical representation. The toolset includes a graphical editor that allows the user to compose and edit graphical formulas on a workstation display and a theorem prover that mechanically checks the validity of proofs in the logic. This paper describes the toolset and illustrates its use.
\end{abstract}

\section{Introduction}

Verifying the correctness of the design of a concurrent system is an extremely difficult and challenging task. The complexity of the problem stems mainly from the need to consider all of the possible orderings or interleavings of events that can be generated by different executions of the system. Nevertheless, the problem is very important because many of the most critical real-world systems are concurrent systems.

Temporal logic is an appropriate formalism for reasoning about the relative ordering of events in concurrent systems $[12,13]$. However, ordinary programmers and engineers have found it difficult to reason in temporal logic and to relate temporal logic to their software and hardware designs; consequently, we have seen little use of temporal logic in industrial applications.

To address this problem we have created Graphical Interval Logic and have implemented a toolset to support its use. Specifications in the logic resemble the "back-of-theenvelope" timing diagrams drawn by system designers. These graphical formulas provide a pictorial representation of the relative ordering of events in a concurrent system. The toolset includes a syntax-directed editor that enables the user to construct graphical formulas on a workstation display. The user can store these graphical formulas in a specification database and print the formulas in their graphical representation.

* This research was supported in part by NSF/DARPA grant CCR-9014382.

** The paper was written by L. E. Moser and G. Kutty, who were responsible for the axiomatization of the logic and for the example. L. K. Dillon designed the graphical syntax of the logic and the graphical editor. G. Kutty implemented the graphical editor. Y. S. Ramakrishna devised the decision procedure and implemented the theorem prover. The original concept of Graphical Interval Logic is due to P. M. Melliar-Smith. 
Underlying the graphical representation is a formal mathematical logic. We have developed an axiomatization for the logic and have demonstrated that it is sound and complete with respect to the class of discrete linear orders [11]. The logic is equivalent in expressiveness to propositional temporal logic with the until operator but without the next operator. We have also developed an automata-theoretic decision procedure for deciding satisfiability of formulas in the logic [18]. As far as we are aware, this is the only known decision procedure of elementary complexity for an interval logic. We have implemented a tableau-based version of the decision procedure, which forms the core of our theorem prover.

Specifications in Graphical Interval Logic are typically structured as a hierarchy of specifications, exploiting composition and abstraction. Verification in such a hierarchy requires a demonstration for each adjacent pair of levels that the specifications at the more concrete level imply the specifications at the more abstract level. The feasibility of this demonstration depends on appropriate use of abstraction and on small proof steps. We consider such verification to be an important part of the design process; experience has shown that, without rigorous mechanical checking, it is difficult to write specifications that do not contain mistakes.

Our approach to controlling the complexity of the verification process is to have the human work in a symbiotic relationship with the theorem prover. Using the graphical editor, the human creates specifications that are the axioms of a theory for the system being modelled. Working in that theory and the underlying logic, the buman creates a theorem and its proof using the graphical editor and then invokes the theorem prover to check the validity of the proof. If the proof is invalid, a counterexample is generated to aid the human in revising and correcting the proof. Creating and/or revising a proof may involve retrieving graphical formulas from the specification database, modifying those specifications, and constructing new specifications using the editor.

The remainder of this paper is organized as follows. In Section 2 we discuss other verification systems that employ a graphical representation or that incorporate support for temporal logic. We then provide a brief outline of Graphical Interval Logic in Section 3. The graphical editor is described in Section 4 and the theorem prover in Section 5. In Section 6 we give an example of the use of the tools in specifying and verifying the two-phase commit protocol, and in Section 7 we present experimental results from the theorem prover for this example application. Section 8 contains concluding remarks.

\section{Related Work}

Graphical representations have been widely used in hardware and software designs of computer systems. However, they have usually lacked a rigorous formal basis and have not been considered an integral part of the verification process. Noteworthy exceptions are the statechart visual formalism of Harel, which has evolved into a working environment for complex reactive systems [7], and the $\forall$-automata formalism of Manna and Pnueli [14]. Both of these formalisms provide a graphical representation of temporal properties, but are oriented toward depiction of states and state transitions rather than toward depiction of the time line and the evolution of properties in time, as in Graphical Interval Logic. 
Procedures for checking the satisfiability of formulas in temporal logic have been implemented by many researchers, both as free-standing decision procedures and within general-purpose theorem provers. For example, Propositional Temporal Logic [13] and Interval Temporal Logic [15] have both been encoded in $\mathrm{ML}$, the meta-language of the higher-order logic theorem prover HOL [8]. We likewise plan to incorporate Graphical Interval Logic into a general-purpose theorem prover.

Niemelä and Tuominen [17] have implemented a system, called the Helsinki Logic Machine (HLM), which is based on a semantic tableau decision procedure for dynamic logic. Decision procedures for temporal logics are realized by translating temporal logic formulas into formulas of dynamic logic and using the decision procedure for dynamic logic.

In [9] Kono described two theorem provers for Moszkowski's Interval Temporal Logic [15]. One of these theorem provers employs a deterministic tableau expansion and a binary decision tree representation of terms, while the other is based on the generation of an automaton and uses Binary Decision Diagrams. Interval Temporal Logic involves the next and chop operators and, thus, is more expressive than Graphical Interval Logic. To reduce the cost of the decision process, Kono has restricted the logic to finite intervals and to formulas without the chop operator in the scope of a negation.

Clarke [4] and others have implemented a variety of tools for model checking in Computation Tree Logic (CTL), a branching-time temporal logic. Model checking in CTL is computationally less expensive than satisfiability checking in linear-time temporal logics, such as Graphical Interval Logic. Unfortunately, the computational cost advantages of CTL model checking do not extend to the general-purpose theorem proving needed for design verification. Recently, Burch, Clarke and others [3] have used a model checking algorithm for the Mu-Calculus based on Binary Decision Diagrams to obtain efficient decision procedures for CTL model checking and satisfiability of linear-time temporal logic formulas.

In [19] Schlör and Damm describe a graphical specification language, called Timing Diagrams, and an approach similar to ours. Specifications resemble traditional informal timing diagrams and are created using a graphical editor. The language is carefully restricted so that it can be compiled into a linear-time temporal logic related to CTL. Model checking for the logic uses computationally efficient CTL algorithms.

The TIMELOGIC system of Koomen [10], based on Allen's interval calculus [1], defines operations on intervals and relationships between intervals, rather than values of predicates within intervals as in Graphical Interval Logic. Koomen's system is an intervalbased forward chaining inference engine and database manager of temporal constraints.

\section{Graphical Interval Logic}

We now present a brief overview of Graphical Interval Logic; details of the syntax and model-theoretic semantics of the logic can be found in [6]. The logic is a linear-time temporal logic that is modelled on the class of $\omega$-sequences of states with a valuation function that defines for each index $i$ the propositions that are true in state $i$. The basic construct of the logic is the interval, which provides a context within which properties are asserted to hold. An interval represents a sequence of states of a computation; the progression of states in time is shown using the horizontal dimension. Graphical Interval 
Logic formulas constrain each computation individually and must be satisfied by every such computation.

Graphical formulas are read from top to bottom and left to right, starting with the topmost interval, which represents an entire computation. An interval is constructed by specifying two search patterns: one to locate the left endpoint of the interval and the other to locate its right endpoint. A search pattern consists of a sequence of searches; the state located by one search is the state at which the next search begins. A search within a search pattern locates the first state at which its target formula holds. An interval begins with the state located by the first search pattern and extends up to but does not include the state located by the second search pattern.

To assert that a formula holds at the first state in an interval, the formula is drawn left-justified below the left endpoint of the interval. For example,

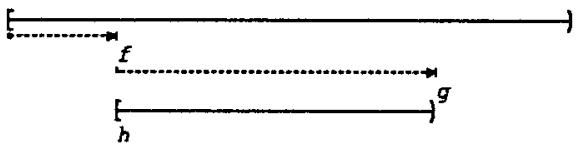

asserts that $h$ holds at the first state in the interval that begins with the first state at which $f$ holds and ends just prior to the next state at which $g$ holds.

To express an invariant (henceforth) property that holds throughout an interval, the formula that is asserted to be invariant is positioned below the interval and indented to the right of the bracket that delimits its start. For example,

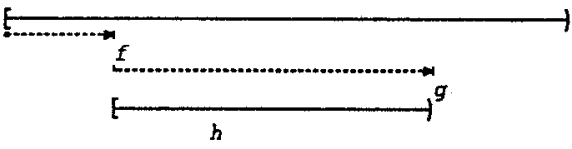

asserts that $h$ holds at every state in the interval that begins with the first state at which $f$ holds and extends up to, but does not include, the next state at which $g$ holds. Temporal expressions that are invariant over the entire computation are indented beneath the topmost interval.

To express an eventuality property, a diamond $\diamond$ is placed on the interval, with the eventuality property left-justified below the diamond. For example,

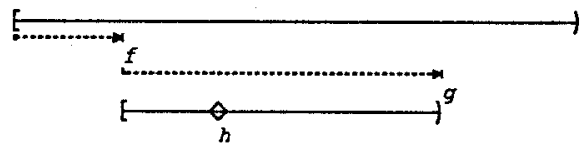

asserts that $h$ holds at some state in the interval that begins with the first state at which $f$ holds and extends up to, but does not include, the next state at which $g$ holds. The subformulas $f, g$ and $h$ in the above examples may be state predicates or more complex interval formulas.

If the target formula of a search does not hold at any state in the future of the state at which the search begins, the search to the formula fails. If either of the searches for the left or right endpoints of an interval fails or, if the state located by the search for 
the right endpoint precedes or coincides with the state located by the search for the left endpoint, the interval cannot be constructed. If the interval cannot be constructed, the interval formula is deemed to hold vacuously.

Formulas can be combined using standard logical infix operators laid out vertically. In vertical layout a conjunction is indicated by stacking the formulas below one another without the conjunction operator. Braces are used to disambiguate formulas.

The single-arrow searches and single-line intervals in the previous examples are referred to as weak searches and weak intervals, respectively. The logic also provides as derived constructs strong searches, denoted by double arrows, and strong intervals, denoted by double lines as, for example, in

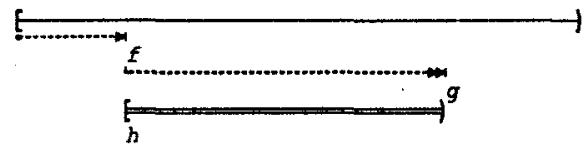

Here the strong search expresses the requirement that the search to $g$ must not fail. More specifically, it requires that the search to $g$ succeeds unless the weak search to $f$ fails. The strong interval requires that the interval is non-empty provided that the searches to $f$ and $g$ do not fail.

\section{The Graphical Editor}

We now briefly describe the graphical editor that facilitates the interactive generation and manipulation of Graphical Interval Logic formulas. The editor enables the user to draw formulas by invoking high-level editing operations based on the constructs of the logic. As a syntax-directed editor, it ensures that the formulas drawn by the user are syntactically correct. The graphical editor also acts as a front-end to the theorem prover.

The menu-and-button interface of the editor allows the user to create and edit graphical formulas and to compose them into more complex formulas. For example, to create a new formula the user first selects the new formula button, which provides a context interval and a box representing a formula that has yet to be defined.

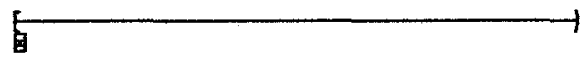

The box is automatically positioned below the left endpoint of the context interval to indicate an initial formula. To denote an invariant or eventuality property, the user would select the pending box and click on the $\square$ or $\diamond$ button.

Instead, the user clicks on the interval button, resulting in a generic interval, and positions the interval with mouse clicks for its left and right endpoints. By clicking for the right endpoint to the right of the end of the context interval, the user indicates a search to the end of the context.

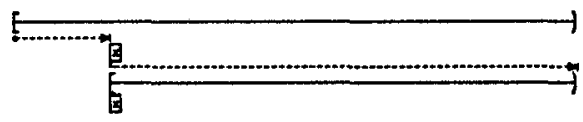


The formula now contains two pending boxes, one for the target of the left search and one for the property of the interval. The user may select either of these pending boxes to expand next. In this example, the user selects the pending box for the left search predicate and then the text button to type in the state predicate $f$.

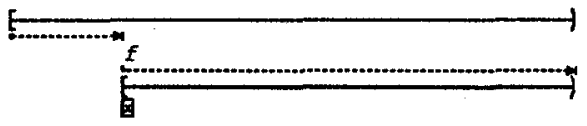

Next the user selects the pending box for the property of the interval and clicks on the $\diamond$ button to indicate an eventuality.

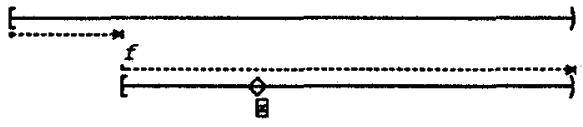

The user then selects the pending box for the property of the $\diamond$ and clicks on the $V$ button, which expands the pending box into a disjunction.

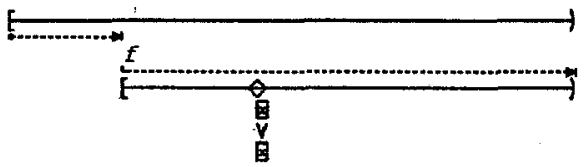

The user could elaborate either of the two pending boxes into an invariant, an eventuality, or a nested interval formula. In this example, however, the user selects each pending box in turn, clicks on the text button and then types in the state predicates $g$ and $h$ to produce the desired formula.

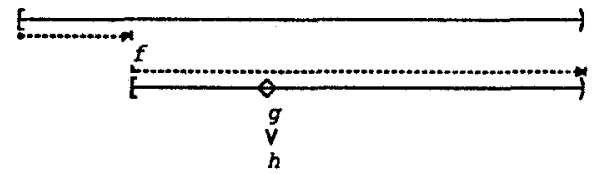

The editor provides capabilities for automatically replacing formulas with other formulas, resizing formulas to suit the context length, etc. In case a formula does not fit into the space allotted, an error is indicated by highlighting the formula. The user may then resize the context length or the search arrows to enable the formula to be drawn correctly. All subformulas of the formula are automatically resized to scale.

The graphical syntax of the logic is defined in terms of an attribute grammar that specifies the set of attributed syntax trees that correspond to "legal" formulas. These trees are used solely as internal representations; the user only interacts with the formulas in their graphical representation. Attributes within these trees give the relative sizes of search lines and interval contexts that allow the formulas to be drawn on the screen. The theorem prover makes use of a simplified representation of the attributed syntax trees produced by the editor. 
As a formula is constructed, the corresponding attributed syntax tree is built incrementally and the attributes that carry information about the graphical representation of the formula are determined. Initially, the formulas generated by the editor contain unexpanded boxes that are expanded into legal formulas during subsequent editing. Each expansion of a box corresponds to a production in the grammar. The consistency of the attribute values for a tree constructed using the editor ensures that the formulas are syntactically correct.

The graphical editor has been implemented in Common Lisp using the Garnet graphics toolkit [16], which runs within the $\mathrm{X}$ window system. The editor uses Garnet functions to generate postscript output. All of the formulas in this paper were produced directly by the graphical editor. Graphical formulas can be stored in and retrieved from a specification database held in Unix files. At present, formulas can only be stored and retrieved by name. We plan to provide a more general querying capability that will allow searching for particular predicates and subformulas.

\section{The Theorem Prover}

Theorem proving in any temporal logic that subsumes propositional calculus is at least NP-hard and, due to the greater expressiveness of most temporal logics, is usually much harder, being at least PSPACE-hard. Our approach to controlling the complexity of the verification process is to have the human work closely in conjunction with the theorem prover. Thus, the human creates the proof using the graphical editor and then invokes the theorem prover to check the validity of the proof.

To prove a theorem $T$ the human selects a subset of the axioms and previously proved lemmas and theorems as the premises $P_{1}, \ldots, P_{n}$ of the proof. It is the responsibility of the human to select a set of premises sufficient to establish the theorem but small enough to keep the proof time reasonable; intermediate lemmas and theorems may be needed. To establish the validity of the proof the human invokes the theorem prover to check the validity of the formula $P_{1} \wedge \ldots \wedge P_{n} \Rightarrow T$. The theorem prover, which is a refutation prover, takes the negation of this formula and searches for a satisfying model. If such a model is found, it is a counterexample to the formula and the purported proof fails. If no such satisfying model exists, the formula is valid and the purported proof is indeed a proof.

The theorem prover exploits the fact that Graphical Interval Logic, in its propositional version, is decidable. As for most temporal logics, a decision procedure may be given as an automata-theoretic method, in which form it is presented in [18]. The decision procedure essentially consists of first reducing the formula into an "equivalent" Büchi automaton and then checking the emptiness of the language of the resulting automaton. The first step involves a double exponential blow-up, i.e. given a formula of size $n$ and depth $k$, its Büchi automaton can have $2^{O\left(n^{*}\right)}$ states. The second step is linear in the number of states of the automaton. In general, it is necessary to construct the full automaton to determine if a satisfying model exists.

The main novelty of our method stems from the need to deal with nested interval contexts, which limit the scope of a temporal formula to its outer interval modalities. This is especially interesting when the temporal formula involves eventualities that are bounded within contexts that may be nested arbitrarily deeply. In [18] we introduce a 
well-founded recursive reduction operation on a formula in a given state of the automaton, which generates a set of simpler conditions that must be satisfied. Bounded eventualities require a specialized transition condition that prevent outer intervals from collapsing (or ending) before nested eventualities are fullfilled.

The automata-theoretic method has been refined into a more traditional tableau method [20], which has lower average-case time and space requirements and which is the basis of our theorem prover. The tableau method is essentially an incremental implementation of the automata method in which unreachable states are never explored. Basically, it produces a compact form of the model-checking automaton by merging states and edges of the automaton. States are merged if for each input there are corresponding transitions from those states to the same state. Edges from a given state are merged if for several inputs there are transitions from that state to the same state.

The tableau procedure constructs a tableau for the formula whose satisfiability is being checked. The tableau is essentially a directed bipartite graph with two types of nodes: state nodes and prestate nodes. The nodes of the graph represent sets of formulas (semantic subformulas of the formula being verified). State nodes have a unique successor, whereas prestate nodes in general do not. For every state node, the corresponding successor prestate node contains the set of conditions that any future following the state must satisfy in order to be accepted.

The tableau procedure also checks for the existence of paths in the graph such that, whenever a prestate node is crossed that contains an eventuality condition, that eventuality is satisfied at some point in the future. The sequence of state nodes that constitute such a path, when projected down to the primitive propositions and their negations, is a satisfying model for the formula. If no such path exists, the procedure terminates after having eliminated the initial node of the tableau and the proof is valid. If such a path is found, that path is a counterexample to the purported proof.

In the event that a proof fails, the graphical interface can be used to display a counterexample model. We are currently investigating the possibility of using this counterexample model to realign the states of the graphical formulas that constitute the invalid proof to assist the user in revising and correcting it.

When using quantified formulas in a proof, the user must supply instances of existentially quantified variables. Thus, the theorem prover is a ground-term prover that operates on formulas after they have been reduced to ground terms, i.e. Skolemized. The Skolemization process is currently manual, but will be automated when the tools are integrated into a more extensive verification system.

As an enhancement to the theorem prover we plan to investigate a technique that will allow the user to identify states in different formulas that are the same state and to position these states accordingly by aligning them vertically. The aim of this userprovided correspondence is to simplify the mechanical proof and, thus, to reduce its cost. It may, however, introduce additional proof obligations that must be discharged.

The tableau procedure has been implemented in Common Lisp on Sun 4 Sparcstations. We currently plan to integrate the decision procedure into a full-scale verification system, such as EHDM [5], which includes decision procedures for propositional calculus and Presburger arithmetic, as well as a Skolemizer and facilities for naming, type checking and modularization. 


\section{An Example Application}

To illustrate the use of the Graphical Interval Logic toolset, we now consider the twophase commit protocol [2]. This protocol is used to commit distributed database transactions. A transaction has the characteristic of being atomic, which means that either it completes successfully and its modifications are available to all of the other transactions, or it aborts and has no effect on the database. The two-phase commit protocol guarantees that either all of the participants in the transaction commit or that all of them abort. The protocol operates as follows:

- The coordinator polls all of the participants, requesting them to vote either to commit or to abort.

- When a participant is polled, it responds with its vote. If the participant votes to abort, it aborts the transaction immediately.

- If all of the participants vote to commit, the coordinator makes the decision to commit; otherwise, the coordinator makes the decision to abort.

- The coordinator informs all of the participants of its decision.

- Each participant that votes to commit must wait for the decision from the coordinator and then commits or aborts as decided by the coordinator.

The coordinator and the participants communicate by message passing, which we assume to be reliable; however, to simplify the example we have abstracted away the communication mechanisms. We assume further that the coordinator and the participants do not fail during the operation of the protocol. In the following, we use the variables $t, s, \ldots$ to denote transactions, $i, j, \ldots$ to denote participants and $x, y, \ldots$ to denote decision values, which are either $c$ to commit a transaction or $a$ to abort it. We use the following predicates.

- poll $(t)$ denotes that the coordinator is polling the participants in transaction $t$.

- vote $(t, i, x)$ denotes that participant $i$ is voting to commit or to abort transaction $t$.

- decide $(t, x)$ states that the coordinator is informing the participants about its decision to commit or to abort transaction $t$.

- committed $(t, i)$ denotes that participant $i$ has committed transaction $t$.

- aborted $(t, i)$ denotes that participant $i$ has aborted transaction $t$.

The specifications for the two-phase commit protocol presented below can be regarded as a definition of the functional behavior of the participants and the coordinator or, alternatively, as a set of requirements imposed on their implementation. We have checked that these specifications contain no redundancies. From the specifications we subsequently derive a set of theorems. These theorems correspond to a more abstract level of specification requiring consistency of commitment but hiding the use of the twophase commit protocol.

In these specifications all free variables are assumed to be universally quantified. We consider the case in which there are two participants. The general case in which there are more than two participants requires a proof by mathematical induction. Specifications 11 and 12 would then involve quantification on the participants. The only proofs that depend on these specifications and that would be affected by the induction are the proofs of Lemmas 3 and 4. 


\subsection{Specifications}

\section{Initial Conditions}

Specification 1. At start-up no actions are taken.

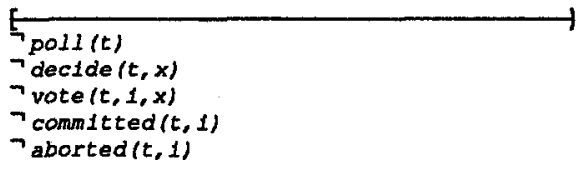

\section{Participants}

Specification 2. If the coordinator polls the participants in a transaction, then each participant will vote on that transaction.

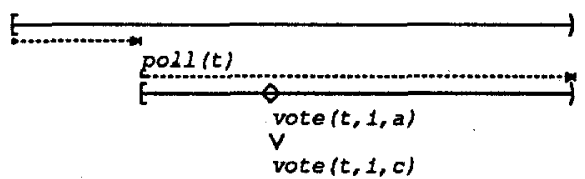

Specification 3. A participant votes on a transaction only if it was previously polled by the coordinator about that transaction.

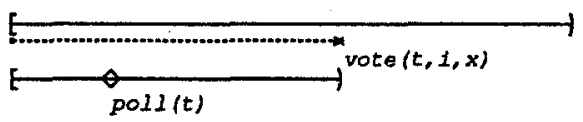

Specification 4. A vote by a participant to abort a transaction precludes a vote by that participant to commit the transaction and vice versa.

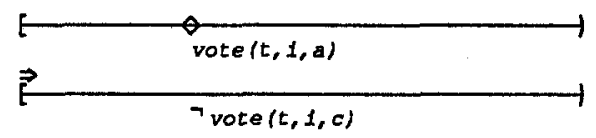

Specification 5. If the coordinator decides to commit a transaction, then every participant acts on that decision and remains committed to that decision.

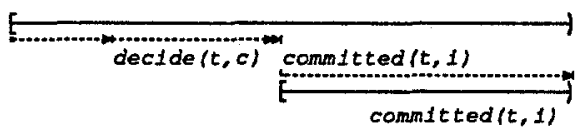

Specification 6. A participant commits a transaction only if the coordinator has previously decided to commit it.

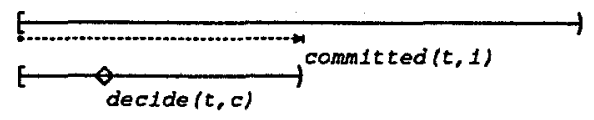


Specification 7. A participant aborts a transaction if that participant has voted to abort it or if the coordinator has decided to abort it.

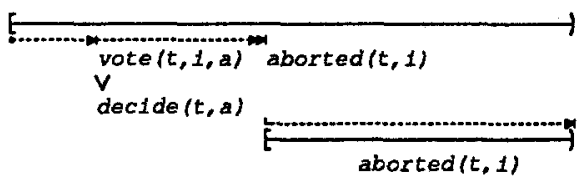

Specification 8. A participant can abort a transaction only under the above conditions.

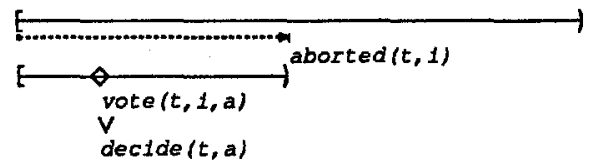

\section{Coordinator}

Specification 9. If the coordinator decides on a transaction, then each of the participants previously voted on that transaction.

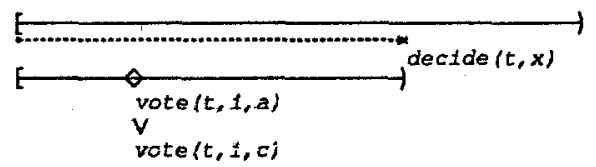

Specification 10. If any participant votes to abort a transaction, then the coordinator will decide to abort it.

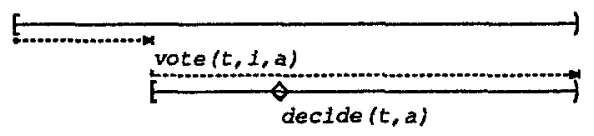

Specification 11. If the coordinator decides to abort a transaction, then one of the participants has previously voted to abort it.

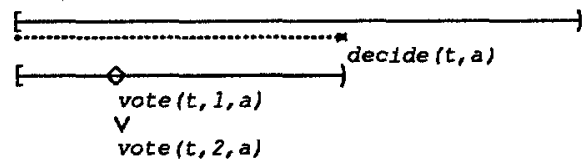

Specification 12. If both of the participants vote to commit a transaction, then the coordinator will decide to commit it.

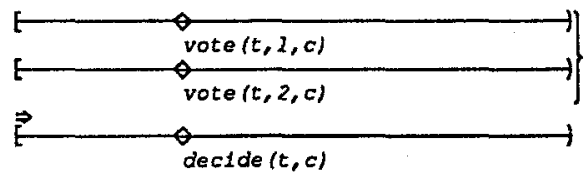


Specification 13. If the coordinator decides to commit a transaction, then each of the participants previously voted to commit it.

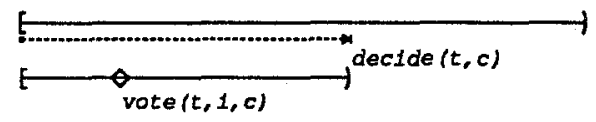

\subsection{Lemmas and Theorems}

We now present some lemmas and theorems derived from the specifications for the twophase commit protocol. Lemmas 1 and 3 and Theorems 4 and 5 provide safety properties, while Lemma 2 and Theorems 1, 2 and 3 provide liveness properties. Each of these lemmas and theorems has been proved from the specifications using the theorem prover. We exhibit one such proof.

Lemma 1. The coordinator does not decide on a transaction before polling the participants in that transaction.

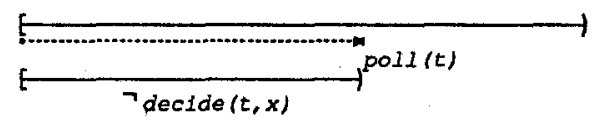

Lemma 2. If the coordinator polls the participants in a transaction, then the coordinator will decide to abort or to commit that transaction.

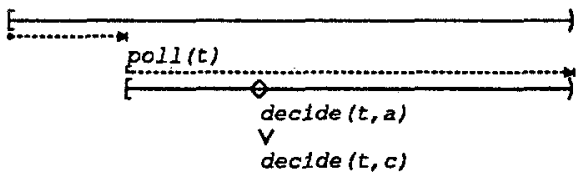

Lemma 3. A decision by the coordinator to abort a transaction precludes a decision to commit and vice versa.

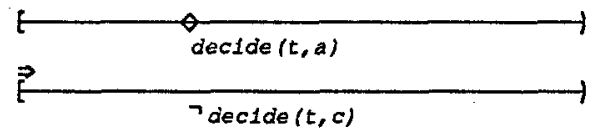

Theorem 1. Each participant in a transaction either commits the transaction or aborts the transaction.

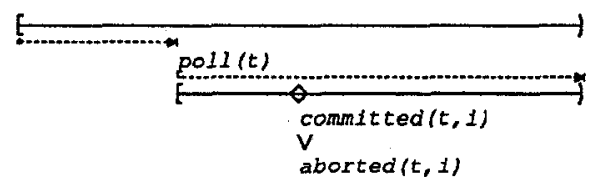

Theorem 2. If one participant commits a transaction, then the other participants will also commit it.

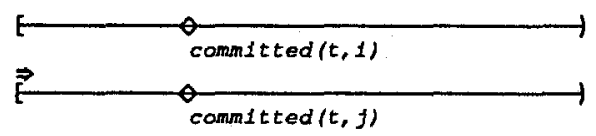


Theorem 3. If one participant aborts a transaction, then the other participants will also abort it.

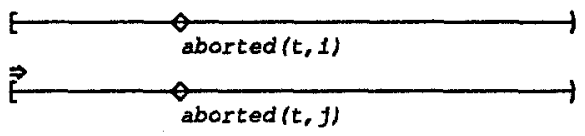

Theorem 4. A decision by a participant to commit is irrevocable.

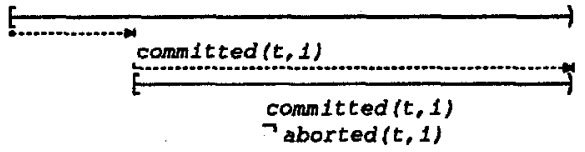

Theorem 5. A decision by a participant to abort is irrevocable.

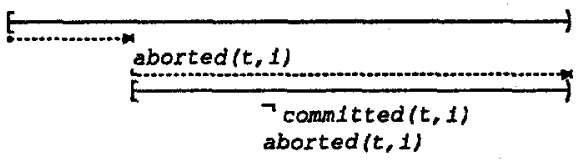

The proof of Theorem 1 is shown below as it is submitted by the user to the theorem prover. The premises of the proof are Lemma 2, Specification 5 and Specification 7 , which appear in the antecedent of the implication. The theorem to be proved, namely Theorem 1, appears in the consequent of the implication. The user submits the proof to the theorem prover as it is shown in its graphical representation.

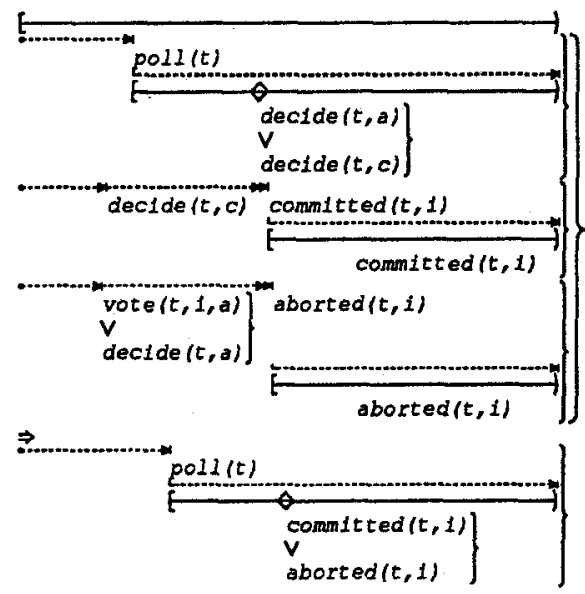

In creating this proof the user employs the following reasoning. If the coordinator polls the participants, then it will decide to abort or commit the transaction. If the coordinator decides to commit, then each participant will commit whereas, if the coordinator decides to abort, then each participant will abort. Consequently, if the coordinator polls the participants, then each participant will commit or abort the transaction. 
Suppose now that the user had constructed the specification below in place of Specification 5 . Both versions of the specification require that committed $(t, i)$ occur after or concurrent with decide $(t, c)$; however, the version below does not require committed $(t, i)$ to remain true once it becomes true.

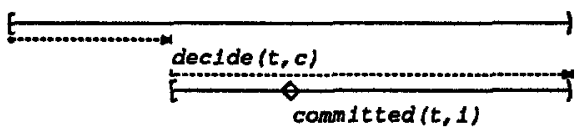

In an attempt to prove Theorem 1 using this version of the specification (together with Lemma 2 and Specification 7), the user discovers with the aid of the theorem prover that the proof is invalid. The graphical interface displays to the user the following counterexample model. The unshaded rectangles indicate the initial section of the model, and the shaded rectangle indicates the infinitely repeated periodic section of the model. Primitive propositions that don't appear in negated or unnegated form take either value true or false.
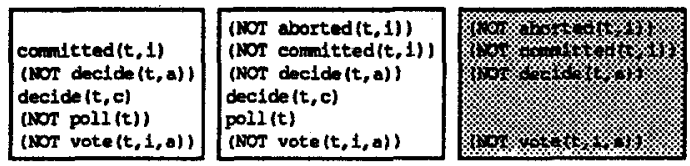

The counterexample model shows the user that the state at which committed $(t, i)$ is true could have occurred before the state at which poll $(t)$ is true rather than concurrent with or after that state. Examination of the counterexample model assists the user in revising and correcting the specification and the proof. Note that the theorem prover considers only the set of formulas selected by the user in creating the proof.

\section{Experimental Results}

The time required for the human to construct specifications and proofs using this methodology depends, of course, on the experience of the human and on the complexity of the system being designed. Construction of the specifications and proofs for the two-phase commit protocol example given above took several man-days, spread over several weeks. Several iterations were required to achieve appropriate specifications.

The computation time required for the proofs for the two-phase commit protocol is given in Table 1. The number of states in the tableau at the end of the first step of the procedure is a rough indication of the complexity of the proof. The shape of the tableau can provide useful clues as to the "content" of a proof. A tableau that is short and bushy indicates that the proof is combinatorial rather than temporal. Deep tableaux, on the other hand, indicate that the proof has large temporal content. Proofs involving case splits are the most bushy, and those involving nested intervals are the deepest. We expect that deep temporal proofs will be decomposed into shorter more manageable ones and, thus, that typical proofs will be bushy rather than deep. 


\begin{tabular}{|c|c|c|c|c|}
\hline \multirow{2}{*}{$\begin{array}{c}\text { Validated } \\
\text { Proof }\end{array}$} & \multirow{2}{*}{$\begin{array}{l}\text { Premises of } \\
\text { the Proof }\end{array}$} & \multicolumn{2}{|c|}{ Size of Tableau } & \multirow{2}{*}{$\begin{array}{c}\text { Time to Check } \\
\text { the Proof }\end{array}$} \\
\hline & & Nodes & Edges & \\
\hline Lemma 1 & $\mathrm{~S} 9, \mathrm{S3}^{2}, \mathrm{S1}^{3}$ & 24 & 32 & $10 \mathrm{~s}$ \\
\hline Lemma 2 & $\mathrm{~S} 2^{2}, \mathrm{~S} 12, \mathrm{~S} 10^{2}, \mathrm{~L}^{2}$ & 673 & 1124 & $2 \mathrm{~m} 28 \mathrm{~s}$ \\
\hline Lemma 3 & $\mathrm{~S}^{2}, \mathrm{~S} 11, \mathrm{~S}^{2} 3^{2}, \mathrm{~S}^{2}$ & 86 & 132 & $24 \mathrm{~s}$ \\
\hline Theorem 1 & $\mathrm{~S} 5, \mathrm{~S} 7, \mathrm{~L} 2$ & 375 & 610 & $46 s$ \\
\hline Theorem 2 & $\mathrm{~S} 5, \mathrm{~S} 6, \mathrm{~S} 1$ & 12 & 16 & $2 s$ \\
\hline Theorem 3 & $\mathrm{~S} 10, \mathrm{~S} 7, \mathrm{~S} 8, \mathrm{~S} 1$ & 52 & 72 & $12 \mathrm{~s}$ \\
\hline & $\mathrm{S} 6, \mathrm{~S} 5, \mathrm{~S} 8, \mathrm{~L} 3, \mathrm{~S} 10, \mathrm{~S} 1$ & 196 & 288 & $47 \mathrm{~s}$ \\
\hline Theorem 5 & $\mathrm{~S} 8, \mathrm{~S} 7, \mathrm{~S} 6, \mathrm{~S} 10, \mathrm{L3}, \mathrm{S} 1$ & 230 & 332 & $1 \mathrm{~m} 10 \mathrm{~s}$ \\
\hline
\end{tabular}

Table 1. Experimental Results for the Two-Phase Commit Protocol. A superscript indicates the number of instances of the formula that are required in the proof. The lemmas that are used as premises of a proof are indicated by $\mathrm{L}$ and the specifications by $\mathrm{S}$. The size of the tableau is the size of the actual data structure constructed; the nodes include both states and prestates.

Several efficiency issues in the implementation of the theorem prover require further exploration. Better heuristics need to be devised for state refinement and choice of formulas to be prouessed in a state. A judicious choice may substantially reduce the running time in many commonly encountered cases. Moreover, our current encoding of states might be improved by using a technique such as Binary Decision Diagrams.

\section{Conclusion}

The toolset for Graphical Interval Logic that we have described includes a graphical syntax-directed editor and an automated theorem prover. The editor enables the user to input specifications and proofs as graphical formulas and subsequently to store and output the graphical formulas. The theorem prover checks the validity of proofs and returns a counterexample if the proof is not valid. We have illustrated the use of the toolset in specifying and verifying properties of the two-phase commit protocol. As this example application indicates, the time required to check the proofs is not unreasonable despite the fact that efficiency was not an overriding concern in this prototype implementation. Recently, we have extended the toolset to include a decision procedure to support verification of real-time properties of concurrent systems.

\section{References}

1. J. F. Allen, "Maintaining knowledge about temporal intervals," Communications of the $A C M 26,11$ (1983), 832-843.

2. P. A. Bernstein, V. Hadzilacos and N. Goodman, Concurrency Control and Recovery in Database Systems, Addison-Wesley, Reading, MA, 1987. 
3. J. R. Burch, E. M. Clarke, K. L. McMillan, D. L. Dill and L. J. Hwang, "Symbolic model checking: $10^{20}$ states and beyond," Information and Computation 98 (1992), 142-170.

4. E. M. Clarke, E. A. Emerson and A. P. Sistla, "Automatic verification of finite-state concurrent systems using temporal logic specifications," ACM Trans. on Programming Languages and Systems 8, 2 (April 1986), 244-263.

5. J. Crow, S. T. Jefferson, R. Lee, P. M. Melliar-Smith, S. Owre, J. M. Rushby, R. L. Schwartz, N. Shankar, R. E. Shostak, F. W. von Henke and A. Whitehurst, "EHDM specification and verification system," Technical Report, SRI International, Computer Science Laboratory, SRI Project 8110, January 1990.

6. L. K. Dillon, G. Kutty, L. E. Moser, P. M. Melliar-Smith and Y. S. Ramakrishna, "Graphical specifications for concurrent software systems," Proc. of 14th Intl. Conf. on Software Engineering, Melbourne, Australia (May 1992), 214-224.

7. D. Harel, H. Lackover, A Naamad, A. Pnueli, M. Politi, R. Sherman, A. Shtull-Trauring and M. Trakhtenbrot, "STATEMATE: A working environment for the development of complex reactive systems," IEEE Trans. on Software Engineering 16, 4 (April 1990), 403-414.

8. The HOL System: Description, SRI International (December 1989).

9. S. Kono, "Automatic verification of interval temporal logic," Proc. of 8th British Colloguium for Theoretical Computer Science (March 1992).

10. J. A. G. M. Koomen, "The TIMELOGIC temporal reasoning system," Technical Report 231, Dept. of Computer Science, The University of Rochester, Rochester, NY, March 1989.

11. G. Kutty, L. E. Moser, P. M. Melliar-Smith, Y. S. Ramakrishna and L. K. Dillon, "Axiomatizations of interval logics," submitted for publication.

12. L. Lamport, "A simple approach to specifying concurrent systems," Communications of the ACM 32, 1 (January 1989), 32-45.

13. Z. Manna and A. Pnueli, "Verification of concurrent programs: The temporal frameworik," Correctness Problem in Computer Science, R. S. Boyer, J. S. Moore (eds.), Academic Press (1981), 215-273.

14. Z. Manna and A. Pnueli, "Specification and verification of concurrent programs by V-automata," Proc. of Conf. on Temporal Logic in Specification, LNCS 398, Altrincham, England (April 1987), 124-187.

15. B. Moszkowski and Z. Manna, "Reasoning in interval temporal logic," Proc. of Workshop on Logics of Programs, LNCS 164, Springer-Verlag, Pittsburgh, PA (June 1983), 371-382.

16. B. A. Myers, D. A. Giuse, R. B. Danneberg, B. VanderZanden, D. S. Kosbie, E. Pervin, A. Mickish and P. Marchal, "Garnet: Comprehensive support for graphical, highly interactive user interfaces," IEEE Computer (November 1990), 71-85.

17. I. Niemelä and H. Tuominen, "Helsinki Logic Machine: A system for logical expertise," Technical Report B1, Helsinki University of Technology, Digital Systems Laboratory, Espoo, Finland, 1987.

18. Y. S. Ramakrishna, L. K. Dillon, L. E. Moser, P. M. Melliar-Smith and G. Kutty, "An automata-theoretic decision procedure for future interval logic," Proc. of Twelfth Conf. on Foundations of Software Technology and Theoretical Computer Science, LNCS 652, SpringerVerlag, New Delhi, India (December 1992), 51-67.

19. R. Schlör and W. Damm, "Specification and verification of system-level hardware designs using timing diagrams," Proc. of European Conf. on Design Automation, Paris, France (February 1993), 518-524.

20. P. Wolper, "The tableau method for temporal logic: An overview," Logique et Analyse, Novelle Série $28^{e}$ Année, 110-111 Numero Special, Automated Reasoning in Non-Classical Logic (June-September 1985), 119-136. 